\title{
Challenges of Applying Optimization Methodology in Industry
}

\author{
Bogdan Filipič \\ Department of Intelligent Systems \\ Jožef Stefan Institute, and \\ Jožef Stefan International Postgraduate School \\ Jamova cesta 39 \\ SI-1000 Ljubljana, Slovenia \\ bogdan.filipic@ijs.si
}

\author{
Tea Tušar \\ Department of Intelligent Systems \\ Jožef Stefan Institute, and \\ Jožef Stefan International Postgraduate School \\ Jamova cesta 39 \\ SI-1000 Ljubljana, Slovenia \\ tea.tusar@ijs.si
}

\begin{abstract}
This presentation starts with two case studies of applying optimization methodology in industry, one involving numerical optimization based on simulation models, and the other combinatorial optimization with specific constraints and objectives. These case studies serve to identify some of the challenges frequently met by solution providers for industrial optimization problems. Based on our experience in applying optimization methodology in industry, we then provide suggestions for dealing with these challenges in order to bridge the gap between academia and industry in optimization.
\end{abstract}

\section{Categories and Subject Descriptors}

I.2.8 [Artificial Intelligence]: Problem Solving, Control Methods, and Search—heuristic methods; G.1.6 [Numerical Analysis]: Optimization-global optimization, constrained optimization; H.4 [Information Systems Applications]: Miscellaneous

\section{General Terms}

Algorithms, Performance, Verification

\section{Keywords}

optimization, evolutionary algorithm, industrial application, parameter tuning, scheduling, constraints

\section{INTRODUCTION}

Optimization problems are regularly faced in various industrial sectors. However, formulating and solving realworld optimization problems, and evaluating the results often prove much more demanding than reported for the textbook problems. The reasons for these challenges are of both technical and subjective nature.

Carrying out academic research in single- and multiobjective stochastic optimization, we have devoted a substantial part of our efforts to transferring the optimization methodology to real-world environments. Most often this was in the form of software solutions to deal with specific optimization problems, either as stand-alone applications or parts of company information systems. Application areas include met-

Copyright is held by the author/owner(s).

GECCO'13 Companion, July 6-10, 2013, Amsterdam, The Netherlands. ACM 978-1-4503-1964-5/13/07. allurgical, automotive, textile and energy sectors. In more than a decade of such collaboration, we have faced a number of challenging situations and developed some strategies for alleviating the difficulties resulting from different views of optimization and its potentials by academia and industry.

This presentation addresses the challenges of applying optimization methodology in industry from the point of view of an academic solution provider. We first present case studies from numerical and combinatorial optimization for industrial customers and then identify typical challenges met in industrial optimization projects. We then suggest the ways of dealing with these challenges in order to bridge the gap between academia and industry in optimization.

\section{CASE STUDIES}

In our applied projects both numerical and combinatorial optimization problems were addressed. A numerical optimization problem handled for several steel plants was tuning of process parameters in continuous casting of steel, while a typical combinatorial optimization problem was production scheduling with specific constraints and objectives.

The optimization task in continuous casting of steel is to set the values of process parameters, such as the casting temperature, speed, and coolant flow rates, so as to maximize the productivity and product quality. The number of parameters is up to thirty and real-world experimental optimization is not feasible as the number of possible settings grows exponentially with the number of considered parameters. Moreover, it would be time consuming and, in case of undesired outcomes, economically unjustified or even dangerous. Therefore, a prerequisite for computer-aided optimization is a reliable numerical model of the casting process. Design of such a simulator required close collaboration of computer and material scientists, and systematic tuning of the simulator to correspond with the real-world casting process. Once this was available, traditional and evolutionary optimization algorithms employing the simulator as a solution evaluator were tested on this task, first in the single-objective form [3], and later considering multiple objectives [4]. The key practical result was improved parameter settings compared to the original manual settings, which reflected in higher quality of the cast steel. Moreover, this application inspired further development the employed optimization methodology to better suit the needs of computationally demanding solution evaluation through parallelization [1].

Production scheduling usually refers to allocation of activ- 
ities to resources over time so that a given objective, such as makespan, is optimized, while resource and temporal constraints are satisfied. However, practical scheduling problems may substantially differ from this template. We dealt with an atypical scheduling problem in a car factory where the task was to schedule interruptions of the production processes (not the processes themselves) in such a manner that the related energy consumption over peak-demand periods was minimized. In addition, the schedules were subject to hard constraints, specifying the maximum number of processes interrupted simultaneously and the shortest time period between two interruptions of a process [2]. We developed a customized evolutionary algorithm that was capable of producing near-optimal schedules and was integrated into the company energy management system. Later we used the experience gained in this project in scheduling flexible offers for energy supply and demand in smart grids [5].

\section{THE CHALLENGES}

Applying optimization methodology in industry is challenging in many ways. Some of the challenges faced in our applied projects are as follows:

- The understanding of optimization and the expectations from it by the solution provider and the customer are often substantially different.

- The solution provider may suffer from insufficient problem domain knowledge, while the customer from not being familiar with the basic concepts of optimization.

- Dealing with an industrial optimization problem rarely starts with a given problem formulation. In contrast, formulating the problem in most cases represents a significant part of the optimization project.

- Not many real-world problems match those from the textbooks. Instead, they involve peculiarities in the form of specific constraints, objectives or additional requirements for result assessment.

- Sometimes, the only way to perform optimization of a system or process is utilizing a simulation model, but a reliable model may be all but trivial to build.

- Optimal values of objectives are typically unknown and this makes the result assessment difficult.

- Even if an optimization approach is demonstrated to be capable of producing beneficial results for the customer, non-technical issues, subjective views or conflicts of interests may prevent its practical use.

\section{BRIDGING THE GAP BETWEEN ACA- DEMIA AND INDUSTRY}

In our experience, considering the following recommendations helps in dealing with the above challenges and contributes to bringing the communities of academic solution providers and industrial customers closer to each other:

- Educating the involved customer personnel about the optimization and acquiring the necessary domain knowledge need to be seen as preparatory steps for an industrial optimization project by the solution provider.
- An interdisciplinary team of experts from the problem domain, optimization methodology, and computer modeling if needed, is a precondition for a successful industrial optimization project.

- Formulating the problem, developing an optimizer, and producing and evaluating the results best work in iterations with gradual improvements until acceptable performance is achieved.

- The measure of success of the optimization project should be clearly defined in terms of the result quality and the optimizer efficiency. In practice, the situation before optimization was applied can serve as a reference point for assessing the results.

- Despite their enthusiasm in optimization methodology, the solution providers should not overemphasize the methodology in interactions with customers, since the customers are interested in solutions to their problems, not the algorithms for finding them.

- Promotion of success stories in academia and industry attracts further interests on both sides and helps bridging the gap between the two communities.

\section{CONCLUSIONS}

We presented two case studies of applying optimization methodology on industrial problems, i.e. model-based optimization of process parameters in metallurgical production, and production scheduling targeted at energy efficiency. They were used to illustrate the challenges of dealing with industrial optimization problems. In our view, understanding these challenges is necessary to successfully cope with them. We also provide recommendations for carrying out industrial optimization projects as a contribution to bridging the gap between academia and industry in optimization.

\section{REFERENCES}

[1] M. Depolli, R. Trobec, and B. Filipič. Asynchronous master-slave parallelization of differential evolution for multiobjective optimization. Evolutionary Computation, 2013, doi: 10.1162/EVCO_a_00076.

[2] B. Filipič. Evolutionary production scheduling in car manufacture. In B. Filipič and J. Šilc, editors, Proceedings of the International Conference on Bioinspired Optimization Methods and their Applications, BIOMA 2004, pages 127-136, Ljubljana, Slovenia, 2004. Jožef Stefan Institute.

[3] B. Filipič and T. Tušar. A comparative study of coolant flow optimization on a steel casting machine. In Proceedings of the the IEEE Congress on Evolutionary Computation, IEEE CEC 2004, Portland, OR, USA, volume 1, pages 569-573, Piscataway, 2004. IEEE.

[4] B. Filipič, T. Tušar, and E. Laitinen. Preliminary numerical experiments in multiobjective optimization of a metallurgical production process. Informatica, 31(2):233-240, 2007.

[5] T. Tušar, E. Dovgan, and B. Filipič. Evolutionary scheduling of flexible offers for balancing electricity supply and demand. In Proceedings of the IEEE Congress on Evolutionary Computation, IEEE CEC 2012, Brisbane, Australia, pages 1212-1219, Danvers, 2012. IEEE. 